\title{
Efficient and Optimal Routing Using Ant Colony Optimization Mechanism for Wireless Sensor Networks
}

\author{
Kavitha $\mathbf{V}^{1}$, Kirupa Ganapathy ${ }^{2}$ \\ ${ }^{1}$ Research Scholar, Saveetha School of Engineering, ChennaiEmail: talk2kavi1983@gmail.com \\ ${ }^{2}$ Associate Professor, Saveetha School of Engineering, SIMATS, Chennai,Email: kirupa1980@ gmail.com
}

\begin{tabular}{l} 
Article Info \\
\hline Article history: \\
Received Apr $7^{\text {th }}, 2018$ \\
Revised Jun $8^{\text {th }}, 2018$ \\
Accepted Jun $20^{\text {th }}, 2018$ \\
\hline
\end{tabular}

\section{Keyword:}

Ant colony optimisation

Bandwidth Integrity

Eternal energy

Premier Nodes

Wireless sensor networks.

\begin{abstract}
Recently more number of routing protocols is discovered for better data routing in Wireless Sensor Network (WSN). However link failures exist in the network due to appearance of low energy nodes, low link gap connectivity while routing, etc. To compute low complexity routes and to minimize the energy consumption a nature bio inspired algorithm Ant Colony Optimization (ACO) mechanism is applied in the sensor networks. An Efficient and Optimal Routing using ACO is proposed. The premium route is determined with sub-premier nodes having high link-gap connectivity factor. The best premier nodes are selected from the sub-premier nodes on basis of bandwidth integrity and eternal energy factors for determining the premium route. The proposed work is validated by comparing the results of other existing techniques. The performance metrics proves that the proposed mechanism exhibits better throughput and delivery rate with low loss rate.
\end{abstract}

\section{Corresponding Author:}

Kavitha V,

Research Scholar,

Saveetha School of Engineering,

Chennai,

talk2kavi1983@gmail.com

\section{Introduction}

Data Transmission from one end to the other end is generally carried out through the routing process. For reliable data transmission, reliable routes are required. Construction of reliable routes in wireless sensor networks is not so easy process since it includes good network properties like delay, energy efficiency, bandwidth, link-gap connectivity, etc., In networks every node is capable of sensing, processing the data, and transmitting to the other. Shortest path routing [Chang \& Tassiulas, 2004] greatly improves the network performance by taking lesser number of hops for transmitting the sensed data.

Most of the IoT applications require rigorous reliability on WSN [Lei et. al, 2016] was implemented since reliability is considered to be one of the most important attributes for industrial operation and applications. Failures in reliability lead to network disaster and it leads to break in carrying out successful network operation results in intolerable serious effects [Koushanfar et al, 2005]. Reliable wireless sensor network can be defined as the network that functions adequately in terms of both connectivity between the sensor nodes and coverage of the targeted nodes i.e. Region of Interest (RoI). Conversely, deploying reliable sensor nodes is a highly challenging task due to random failures of sensor nodes. The sensor nodes gets failed 
due to several factors like depletion in battery level, malfunctions in hardware etc., Therefore the redundant sensor nodes should be made available at anytime.

Ant Colony Optimisation is a swarm intelligence bio inspired algorithm introduced for making the networks more reliable. Various constraints analysis is used to make the network more efficient. In the way, the nodes located in the network and the coverage area between them, energy depletion of each nodes, link gap connectivity among the sensor nodes for data transmission, etc are analysed by applying ACO local and global optimum methods.

\section{Related work}

Several techniques were proposed to improve data routing among sensor nodes. Huge research work was taken for the analysis of routing among sensor nodes and reducing the cost of locating the nodes under different circumstances. Most of the routing protocol doesn't meet the connectivity requirements and leads to the sensor node deployment problem. Some of the routing protocols were discussed here as follows: Energy efficient On-demand Multicast Routing Protocol [Hao et al, 2013] is the primary routing protocol was proposed to improve the network lifetime by removing the energy lacking nodes from multicasting the data packets. The destination driven mechanism is incorporated in the multicasting routing protocol so that the cost of tree construction process gets reduced.

Transmission radius self adjust energy saving routing protocol [Luo et al, 2008] had proposed for minimising the energy usage while sending data packets from source sensor node to the destination sensor node. Here the node reduces the transmission radius at the maximum end for sending the data to the farthest neighbour node. When the node reaches a certain threshold the transmission radius of the node again reduces for lesser energy consumption during transmission. When transmission radius of the node reduces then more packet loss might cause due to insufficient signal strength.

A protocol named RBPC (RREP Broadcast with Passive Clustering) [Hao et al, 2008] was proposed to reduce the route search. Broadcasting route reply packets with expanding rings reduces the route search for many source nodes. This reduces the refrains of unnecessary RREP therefore reduces the control overheads. Asymptotic analyses of minimum scan statistics [Yi, 2009] of node properties such as coverage of nodes, connectivity of nodes, largest edge length of geometric structure and local minimum free geographic. Node Transmission radius is taken as a solution for solving many routing issues that occurred in the network.

To prolong the network lifetime, Ant colony optimisation approach [Hui et al, 2009] is applied, here path delay, node energy and frequency of the node that acts as a router are considered for achieving dynamic and adaptive routing. However there exist energy efficiency problem, in order to overcome the issue Three Pheromones ACO (TPACO) [Lee et al, 2011] was proposed for solving energy efficient coverage for WSN. In this approach, three pheromones such as one local pheromone and two global pheromones were used for finding active sensor nodes per point of interest. Probabilistic sensor identification is applied in local pheromones and using various kinds of sensors (heterogeneous nodes) is applied in global pheromones.

Further, Ant-Colony-Based Scheduling Algorithm (ACO-SA) [Lee \& Lee, 2012] was proposed. This protocol is fully optimised for solving Energy Efficiency (EE) issues by using probability sensor identification model. The algorithm is applied for heterogeneous sensor group since it was a more realistic approach for solving energy efficient coverage problem. Ant Colony Optimization with Three Classes of Ant Transitions (ACO-TCAT) [Liu, 2012] had proposed for decreasing the low-graded solutions. This algorithm reduces the searching range and the connectivity-guaranteed grid coverage issue can be solved using three classes of transition of ants. However, the end to end delay is not considered in this scheduling algorithm. 
Swarm Intelligence (SI) based routing topology was proposed for effective monitoring of link failures and congestion control in WSN [Krishna \& Doja, 2011]. Particle Swarm Optimization (PSO) and Ant Colony Optimization are the SI models for routing the data. SI evaluates particles position and velocity in PSO, pheromone cost and forage success rate in ACO. Self organising networks generally act autonomously for achieving best performance. If number of nodes increases then network size increases and this leads to optimization problem, therefore Non-Dominated Quantum Optimization (NDQO) [Alanis et al, 2014] algorithm was proposed. This algorithm evaluates the valid routes by using Pareto optimality method and minimizes the network complexity as well.

Effective transmission strategy should be achieved based on maximum possible energy balancing (MPEB) and Maximum possible EE (MPEE) [Liu X, 2014]. The effective transmission strategy is done based on the application of ACO mechanism. Here the ant move only one step to complete one trip and heuristic information is not available in the ant transition probability. For load balancing a node deployment scheme was proposed on basis of ACO [Huang et al, 2015]. Group based connection method using group division of nodes was modelled for avoiding blind connection between nodes and this method reduce deployment cost as well by evaluating actual load transmission for critical region.

ACO is applied for improving lifetime for heterogeneous network and to solve combinatorial problems [Lin et al, 2012]. A construction graph is used for denoting the device assignment and the optimal path is chosen based on heuristic information built on the number of connected covers. Local optimal-distance achievement is essential for energy balancing networks therefore optimal distance based transmission mechanism [Liu X, 2015] was proposed. For evaluating lifetime of the network, global optimal distance gain scheme is taken for consideration.

Efficient ACO scheme [Vaishali \& Nighot, 2016] was proposed formobile-sink based WSN. Due to mobile sink general routing protocol requires multiple times of route reconstruction. Here reconstruction of routes is reduced by applying ACO mechanism since single and efficient route is constructed based on heuristic information and the route is considered to be optimal. The NP (Non-polynomial) complete problem is still addressed while deploying the reliable nodes in the network.

Local search heuristic [Deif \& Gadallah, 2017] was applied to reduce the NP hardness complete issue. The nodes are placed at the minimum coverage distance to provide better communication. Optimal-Distancebased Transmission Approach [Liu, 2015] on basis of ACO (ODTA-ACO) was proposed for achieving local optimal distance as well as energy balancing network system. Energy depletion minimisation of sensor nodes can be achieved by acquiring global optimal distance so that low energy is consumed by the sensor nodes for transmission. However the computational complexity in the network gets increased due to this mechanism.

To overcome the shortcomings of the above proposed techniques and to improve the network reliability an efficient and optimal routing protocol is proposed here. To propose a network with higher lifetime, to reduce energy depletion rate and to achieve maximum data transfer rate a scheme named Efficient and Optimal Routing using ACO is implemented. The nodes are located randomly in the network and the premium path from source node to the destination node is constructed via premier nodes for achieving maximum data transfer rate with low energy consumption. 


\section{Proposed method}

The sensor nodes placed in the network leads to frequent link failures while sending data from one node to another due to adjustments in sensor node location. Generally the protocols uses route request message for discovering the routes and route reply message for creating the link between the nodes. However, the control messages create high congestion during data forwarding process. The objective of the work is to implement highly satisfied routes with minimum execution or processing time. Therefore, an Efficient and Optimal Routing using ACO (EOR-ACO) is proposed. By applying Ant Colony Optimisation technique the network congestion can be controlled by means of electing best path. Selecting the nodes with high optimality resource level such as energy and bandwidth is mandate for reducing the overall time consumption of network routing and congestion.

ACO Mechanism: Ant travels from their nest (source) to the places where it can reach for the food (destination). Ant chooses the shortest path in order to reach the destination faster. Beginner ant drops pheromone so that follower ants can take the shortest path easier. In the same way the source node (nest) broadcasts the route request (drop pheromones) towards the destination node (food) in order to find the efficient path. The dropped pheromones gets evaporated in few seconds and it is termed as Time to Live (TTL) i.e. path expiry time. The RREQ packet contains information's like node ID, number of middle nodes in the path, node's energy level, available link time, bandwidth and the data's are required to maintain the routing table that is shown in figure 1. The traditional protocol named DSDV (Destination Sequenced Distance Vector) protocol is considered for routing from source to destination node.

\begin{tabular}{|l|c|c|c|c|c|}
\hline NodeID & Neighbour nodes & Node Energy & Bandwidth & Link duration & TTL \\
\hline
\end{tabular}

Figure 1: Routing Table

The network consists of number of sensor nodes and efficient route should be established between the source and destination node. Premium path is selected using premier nodes and these premier nodes are detected using their optimality resource constraints such as level of energy, delay and bandwidth.

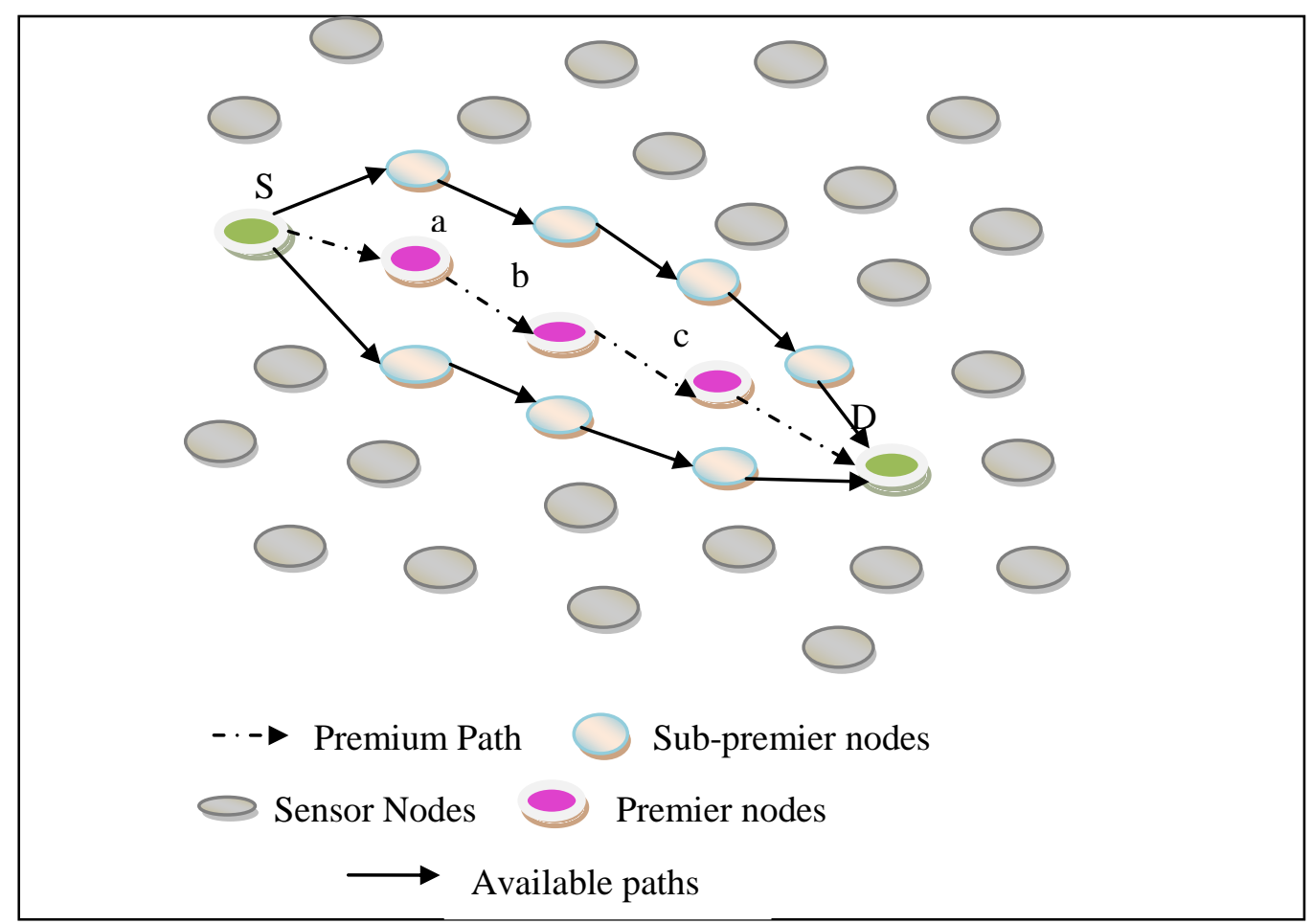

Figure 2: Network architecture of EOR-ACO scheme 
Source node ' $\mathrm{S}$ ' sends data to the desired destination node ' $\mathrm{D}$ ' node by way of Premier Nodes (PN) such as $\mathrm{a}, \mathrm{b}$ and $\mathrm{c}$ which is shown in figure 2 . The network may consists of several premier nodes, if multiple PN present in the routing path, then the best PN is selected among them using the constraints such as residual level of energy and bandwidth. The premium routing path is selected here as S-a-b-c-D.

\subsection{Detection of Premium Route using ACO mechanism}

An efficient route is detected for data transmission by applying ACO mechanism in the network. Source node ' $S$ ' (acts as nest) generates RREQ message and broadcast to the neighbour nodes, neighbour nodes receives RREQ (pheromones) and replies with RREP if it is a destination node (food area) else the RREQ passes until it reaches the destination. The pheromones dropped by the ants can be evaporated in some amount of time and that time is said to be Time to Live (TTL). The level of signal strength of the RREQ message is detected using TTL period. Total existing time of the link can be estimated using the number of RREQ messages. Data can be transmitted using the control messages. The link availability calculation is based upon the signal availability time. Therefore it is calculated using both signal level and the distance between the nodes. Link existing period can be calculated with the estimation of dropped RREQ using the Equation 1.

$$
L_{E t}=\sum \varphi_{a, b}+\left(2 \pi r_{a} * I_{2} o\right)
$$

Where $\mathrm{L}_{\mathrm{Et}}$ represents link existing duration, $\varphi_{\mathrm{a}, \mathrm{b}}$ corresponds to the distance between the present source node ' $a$ ' and its neighbourly located node ' $b$ '; $2 \pi r_{a}{ }^{*} I^{2}{ }_{0}$ represents the average received power from the nodes.

The probability of link existing time with optimal route (EOR-ACO) is calculated using the Equation 2.

$$
P a b=\frac{\varphi_{\mathrm{ab}}^{\alpha} *_{\mu_{\mathrm{ab}}}^{\beta}}{\sum \mathrm{L}_{\mathrm{Et}}}
$$

Where $\mu_{\mathrm{ab}} \rightarrow$ successive RREQ

$\varphi_{\mathrm{ab}} \rightarrow\left(1 / \mathrm{d}_{\mathrm{ab}}\right) \rightarrow \mathrm{d}_{\mathrm{ab}}$ (distance exists between the nodes a and $\left.\mathrm{b}\right)$

$\alpha$ and $\beta \rightarrow$ consideration factor to determine TTL

If TTL gets expired or there is no link exists between the nodes, then the probability of link existing time with optimal route grade $\mathrm{P}_{\mathrm{ab}}=0$. Level of dropped pheromone assessment can be determined by TTL calculation i.e. the routing path for data exists between node to node for communication by passing control messages. $\mathrm{P}_{\mathrm{Th}}$ is the threshold level or reference value for link availability. The reference value can be estimated using equation 3 .

$$
\frac{P_{T h}=\sum \mathrm{L}_{\mathrm{Et}}}{\text { time }}
$$

For optimal routing premier nodes are selected from the sub premier nodes. Premier nodes are picked from other nodes by determining the residual energy. 


\subsection{Premier nodes selection for data transmission phase}

The premier nodes present in the network is selected for reliable and optimal routing. Premier nodes are selected from the sub premier nodes using node constraints such as Bandwidth Integrity (BI) and Eternal Energy Level (EEL). PN is selected among multiple sub premier nodes for efficient and reliable data transmission with minimum execution time. Eternal energy level of the node is determined by considering the balance energy leftover after the consumption of energy for previous data transmission of nodes that exist in the routing path. Bandwidth Integrity (BI) estimation can be done using link-gap analysis. The maximum number of packets that can be sent over a link is said to be bandwidth integrity. Therefore the premier nodes are selected based on the analysis of two factors such as BI and EEL.

The routing table of the network consists of Node ID, Energy level, Bandwidth, Link existing time, Hop_counts, TTL. NodeID is used to identify from which node the control message i.e. RREQ and RREP has received. Energy level specifies the current energy level or residual energy level of the node. Bandwidth estimation includes analysing the amount of link-gap available using equation 4 and calculating the average bandwidth utilisation for particular transmission of information. Low-level link-gap node connectivity is enough for transmitting lesser number of packet transmissions. Bandwidth reference level is set for all the premier and sub premier nodes using equation 5 and the Bandwidth data rate ' $\mathrm{BI}$ less than the $\mathrm{B}_{\mathrm{Th}}$ have chosen for data transmission. Therefore this node factor analysis improves better achievable data rates.

$$
B I=\frac{\text { Number of pkts }(n) \text { sent }}{\text { time taken }} \quad(\text { bps })
$$

The Bandwidth threshold or reference ' $\mathrm{B}_{\mathrm{Th}}$ ' is set by estimating the average packet size used to communicate by the node. The estimation includes channel rate and overall control overheads as well.

$$
B_{T h}=\sum B I
$$

The node with high eternal energy level is considered as one factor for selecting the premier nodes. Eternal energy level analysis is done based on the node drain rate, which is mandate in order to sustain better link quality between the nodes. The nodes with high energy drain rate are kept away from routing so that better communication link can be maintained. Energy threshold is maintained in the network. The drain rate of eternal energy $\left(\mathrm{EE}_{\mathrm{d}}\right)$ can be estimated by measuring the differences between initial energy level $\mathrm{E}_{\mathrm{I}}$ and instant energy level $\mathrm{E}_{\mathrm{i}}$ with respect to time and it is given in equation 6.

$$
E E d=\frac{E_{I}-E_{i}}{t_{c}}
$$

Figure 3 shows the steps in efficient and optical routing using ACO. Reference energy value or energy threshold ' $\mathrm{E}_{\mathrm{Th}}$ ' for all the nodes is set by considering the overall drain rate of eternal energy. Therefore $\mathrm{E}_{\mathrm{Th}}$ can be evaluated by considering the average energy drain rate as in equation 7 .

$$
E_{T}=\operatorname{Avg}\left(E_{d}\right)
$$

Based on node factors BI and EEL the pheromone content or the link gap connectivity among the nodes is updated. Based on the link gap connectivity factor, the premier nodes are chosen from the subpremier nodes for efficient data transmission operation. 


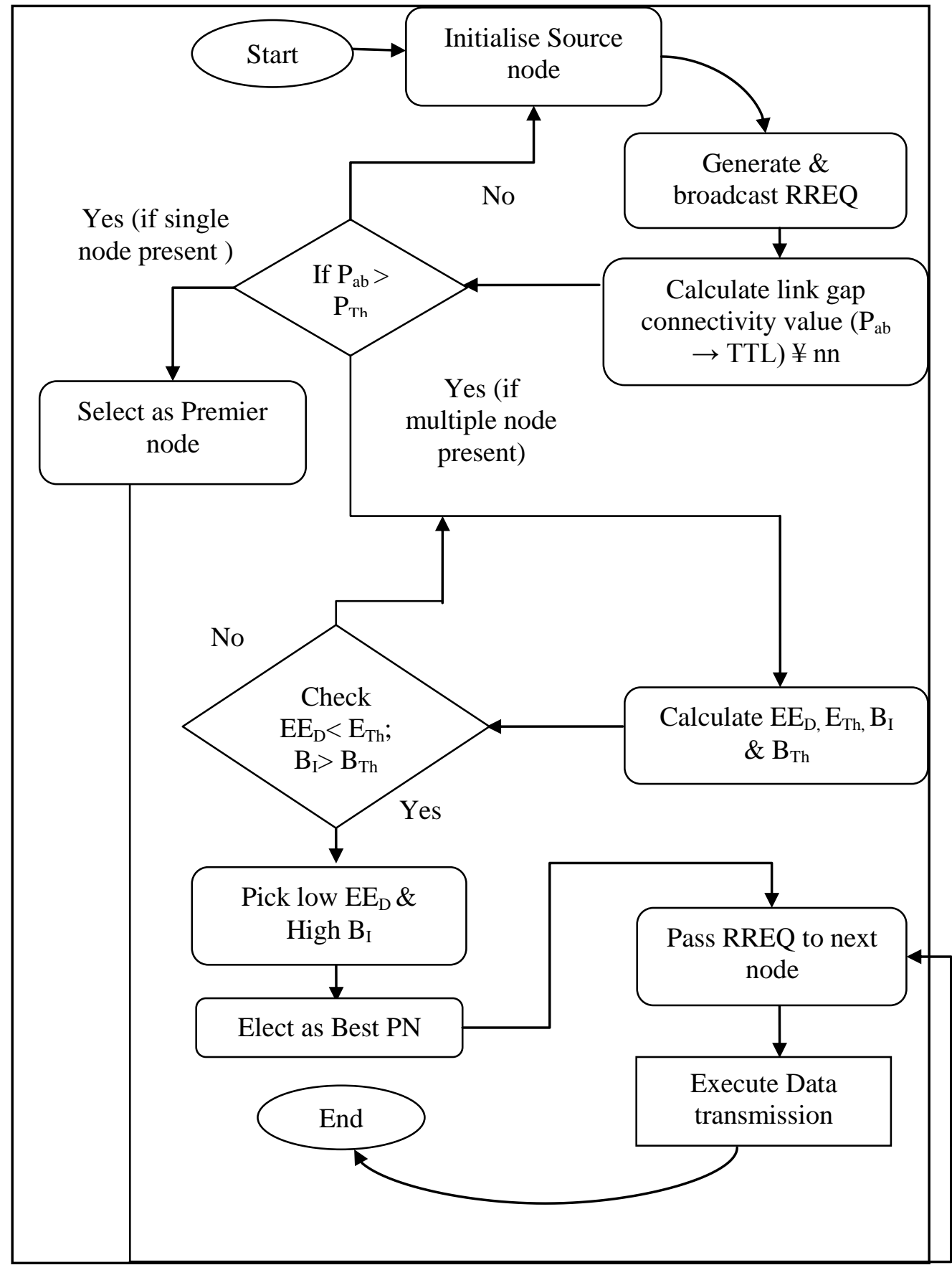

Figure 3: Efficient and optimal routing using ACO 


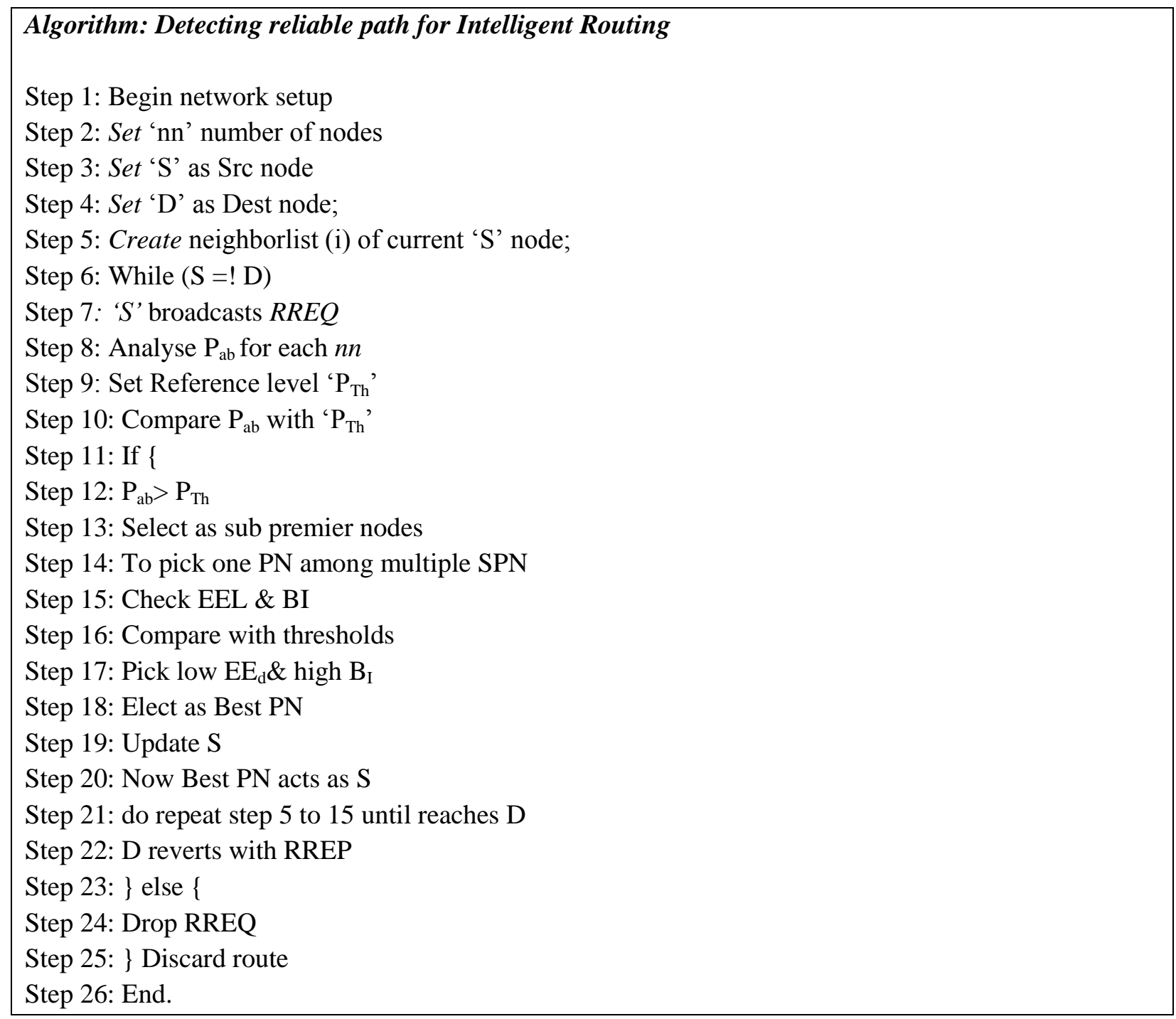

\section{Results and discussion}

To validate the method proposed here, a simulation scenario with 50 nodes deployed and configured as the subterranean wireless network is used with the specifications mentioned in the table 1. Programming in $\mathrm{C}++$ and Object-oriented Tool Command Language (OTCL) is done to determine the locations of various targeted nodes.

The performance of the existing system TPACO is compared with the proposed EOR-ACO system, which is analyzed in this work using simulation results. In order to analyze the performances, the packet delivery rate, bandwidth integrity, throughput, delay and leftover energy are compared through simulations.

Table 1: Simulation parameters

\begin{tabular}{|c|c|}
\hline Parameter & Value \\
\hline Simulation Time & $60 \mathrm{~s}$ \\
\hline Number of Nodes & 100 \\
\hline Routing Protocol & EOR-ACO and TPACO \\
\hline Simulation Area & $800 x 800$ \\
\hline Transmission Range & 250 \\
\hline Antenna Type & Omni Antenna \\
\hline Network Interface Type & WirelessPHY \\
\hline Channel Type & Wireless Channel \\
\hline
\end{tabular}




\subsection{Delivered packet rate}

The rate of packets that sent over to the destination to the rate of packets that sent by the source measures the packet delivery rate. The rate of packet deliveries measurement is given in equation 8 .

$$
P D R=\frac{\sum \text { Pkts Received }}{\sum \text { Pkts Sent }}
$$

'Pkts sent' is represented as number of packets sent by the source node and 'Pkts Received' is represented as number of packets received by the destination for data transmission. The figure 4 shows that the proposed scheme EOR-ACO has better delivery rates of packets at the receiver end compared to the existing method TPACO and ODTA-ACO. Electing the premium nodes from the available nodes in the path makes the network more efficient that in turn reduces packet loss effectively. This leads to achieve better packet rates at the receiver node for the proposed scheme EOR-ACO.

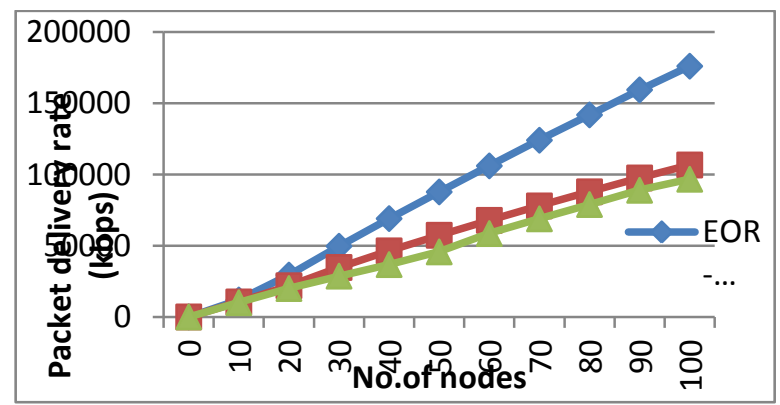

Figure 4: Packet delivery rate

\subsection{Throughput}

Throughput can be defined as the successful transmission of information at the receiver that passed over the network. The overall performance of the network system is measured using the equation 9 .

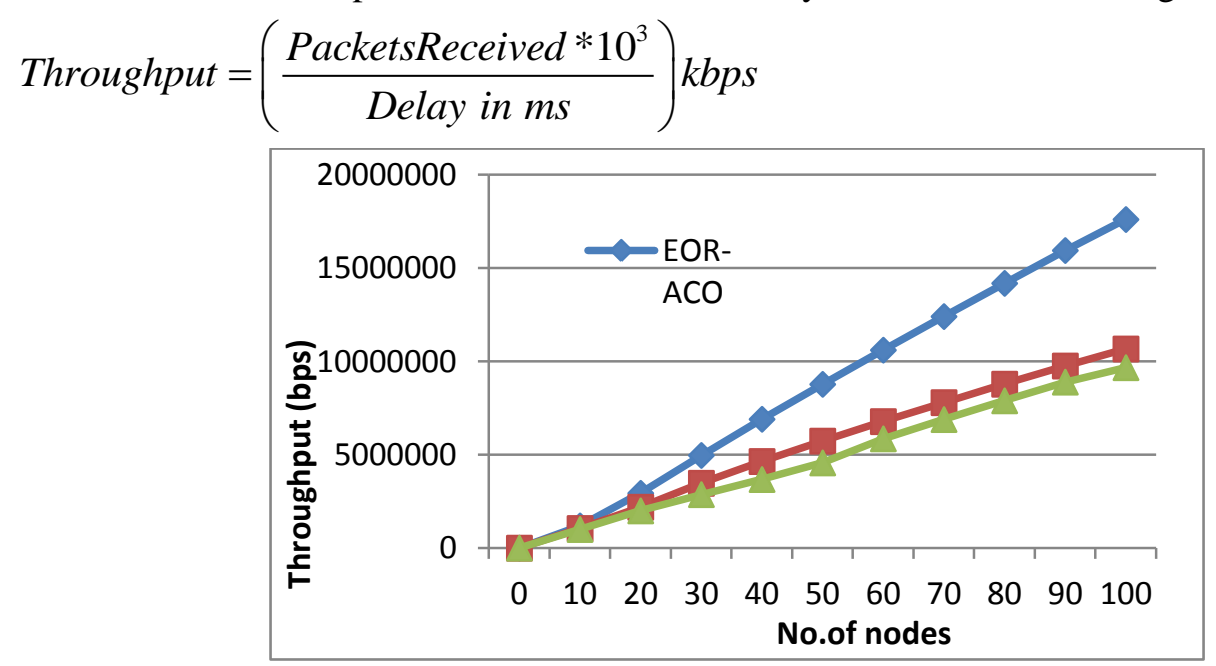

Figure 5: Throughput

Figure 5 shows that performance of the network throughput which is the comparison of both existing and proposed method. The result proved that the throughput for the proposed scheme EOR-ACO is better than the conventional TPACO and ODTA-ACO since delivery rates of proposed is high. Selection of premium route reduces the loss rate therefore throughput gets increased for the proposed EOR-ACO. 


\subsection{Leftover energy}

The remaining amount of energy level present in the sensor node at the current instance of time after processing the data transfer is called as leftover energy or residual energy of the node. This is measured by the difference between the current energy of the node and the initial energy level givento the node.

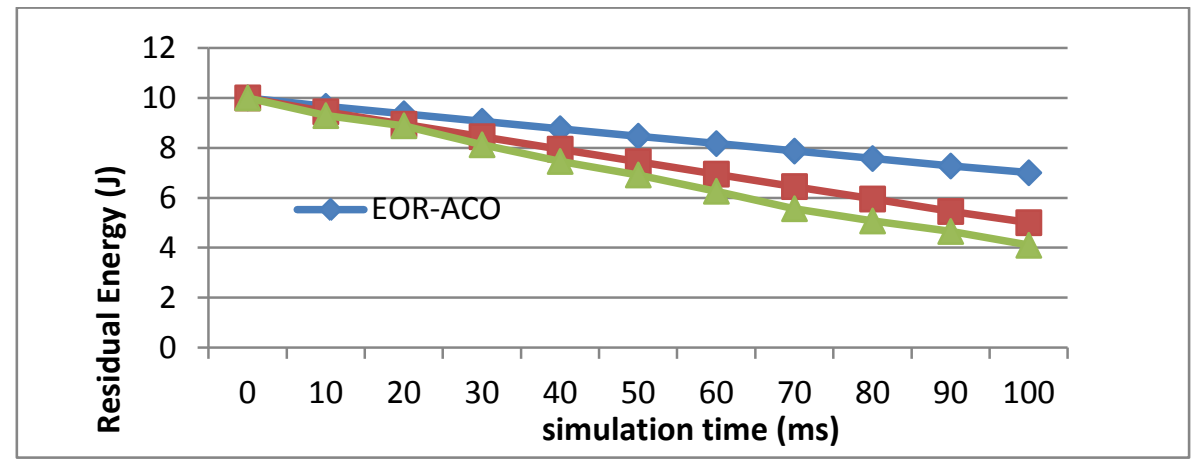

Figure 6: Leftover energy

The figure 6 shows the leftover energy for the conventional TPACO and ODTA-ACO scheme and the proposed method EOR-ACO. Among these schemes the proposed optimal EOR-ACO consumes lesser energy for processing the data. The sensed data's are transmitted over premium nodes which have higher residual energy, therefore the proposed scheme EOR-ACO shows better results in leftover energy compared to conventional cases.

\subsection{Delay}

Delay is measured for the time difference that takes for the network process to complete their data transmission from one end to the other end. The delay can be measured in terms of time difference exists between the received packets and the sent packets. It can be measured by using equation 10, where ' $n$ ' refers to the number of nodes.

$$
\text { Delay }=\frac{\sum_{0}^{n} P k t \text { Recvd Time }- \text { Pkt Send Time }}{n}
$$

The delay for the proposed and conventional schemes is shown in the figure 7. It is clearly shown that the proposed EOR-ACO has better performance in terms of ETE- delay compared to the conventional scheme TPACO and ODTA-ACO. The routes discovered before the expiration of TTL in the proposed EOR-ACO hence the data transmission process is done with lower end-to-end delay. 


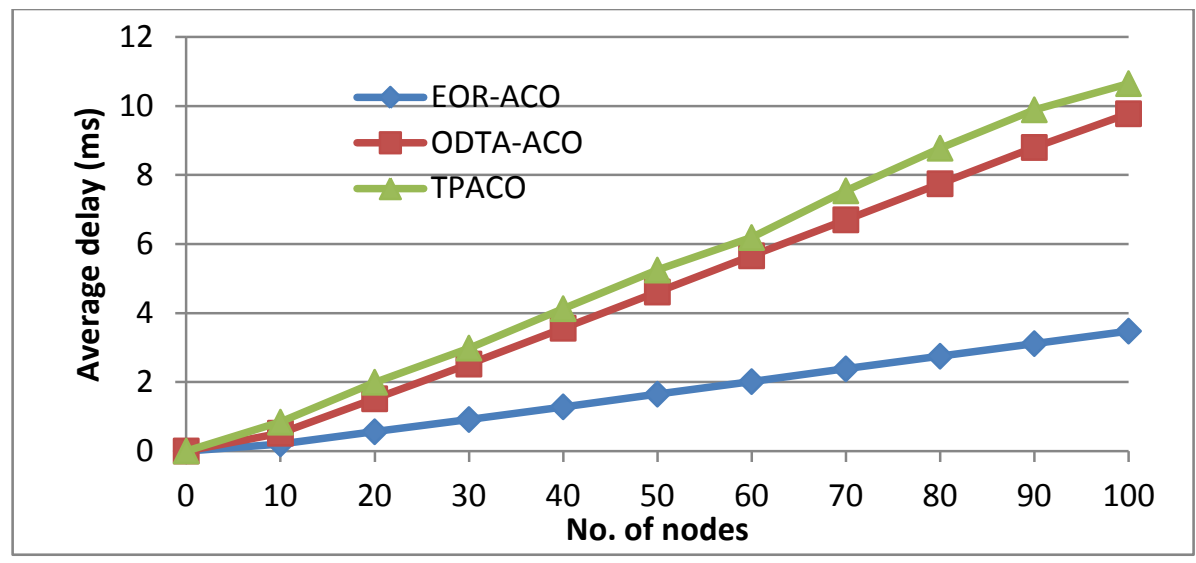

Figure 7: End to End delay

\section{Conclusion}

An Efficient and Optimal Routing using ACO scheme is implemented for improving the network characteristics and its functions. This scheme is proposed to decrease the link failures exist in the network due to appearance of low energy nodes, low link gap connectivity while routing, etc. To compute low complexity routes and to minimize the energy consumption a nature bio inspired algorithm ACO mechanism is applied in the sensor networks. The premium route is determined with the help of located sub-premier nodes by analysing their high link-gap connectivity factor. The best premier nodes are selected from the sub-premier nodes on basis of bandwidth integrity and eternal energy factors. The performance metrics proves that the proposed mechanism exhibits better throughput and delivery rate with low loss rate. The efficiency is achieved up to $30 \%$ for the proposed EOR-ACO compared to the conventional ODTA-ACO and TPACO.

\section{References}

1. Chang, J. H., \& Tassiulas, L. (2004). Maximum lifetime routing in wireless sensor networks. IEEE/ACM Transactions on networking, 12(4), 609-619.

2. L. Lei, Y. Kuang, X. Shen, K. Yang, J. Qiao, and Z. Zhong,Optimal reliability in energy harvesting industrial wireless sensor networks,' IEEE Trans. Wireless Commun., vol. 15, no. 8, pp. 5399_5413, Aug. 2016.'

3. F. Koushanfar, M. Potkonjak and A. Sangiovanni-Vecentelli, "Fault tolerance in wireless sensor networks," in Handbook of Sensor Networks: Compact Wireless and Wired Sensing Systems, 1st ed. Boca Raton, FL, USA: CRC Press, 2005, ch. 36

4. Hao, J., Duan, G., Zhang, B., \& Li, C. (2013, December). An energy-efficient on-demand multicast routing protocol for wireless ad hoc and sensor networks. In Globecom Workshops (GC Wkshps), 2013 IEEE (pp. 4650-4655). IEEE.

5. Luo, D., Zuo, D., \& Yang, X. (2008, October). An energy-saving routing protocol for wireless sensor networks. In Wireless Communications, Networking and Mobile Computing, 2008. WiCOM'08. 4th International Conference on (pp. 1-4). IEEE.

6. Hao, B., \& Li, C. (2010, September). RBPC: A Scalable Routing Protocol for Large Scale Wireless Sensor Networks. In Wireless Communications Networking and Mobile Computing (WiCOM), 2010 6th International Conference on(pp. 1-4). IEEE.

7. Yi, C. W. (2009). A unified analytic framework based on minimum scan statistics for wireless ad hoc and sensor networks. IEEE Transactions on Parallel and Distributed Systems, 20(9), 1233-1245.

8. Hui, X., Zhigang, Z., \& Xueguang, Z. (2009, July). A novel routing protocol in wireless sensor networks based on ant colony optimization. In 2009 international conference on environmental science and information application technology(pp. 646-649). IEEE. 
9. Lee, J. W., Choi, B. S., \& Lee, J. J. (2011). Energy-efficient coverage of wireless sensor networks using ant colony optimization with three types of pheromones. IEEE Transactions on Industrial Informatics, 7(3), 419-427.

10. Lee, J. W., \& Lee, J. J. (2012). Ant-colony-based scheduling algorithm for energy-efficient coverage of WSN. IEEE sensors journal, 12(10), 3036-3046.

11. Liu, X. (2012). Sensor deployment of wireless sensor networks based on ant colony optimization with three classes of ant transitions. IEEE Communications Letters, 16(10), 1604-1607.

12. Krishna, M. B., \& Doja, M. N. (2011). Swarm intelligence-based topology maintenance protocol for wireless sensor networks. IET wireless sensor systems, 1(4), 181-190.

13. Alanis, D., Botsinis, P., Ng, S. X., \& Hanzo, L. (2014). Quantum-assisted routing optimization for self-organizing networks. IEEE Access, 2, 614-632.

14. Liu, X. (2014). A transmission scheme for wireless sensor networks using ant colony optimization with unconventional characteristics. IEEE Communications Letters, 18(7), 1214-1217.

15. Huang, G., Chen, D., \& Liu, X. (2015). A node deployment strategy for blindness avoiding in wireless sensor networks. IEEE Communications Letters, 19(6), 1005-1008.

16. Lin, Y., Zhang, J., Chung, H. S. H., Ip, W. H., Li, Y., \& Shi, Y. H. (2012). An ant colony optimization approach for maximizing the lifetime of heterogeneous wireless sensor networks. IEEE Transactions on Systems, Man, and Cybernetics, Part C (Applications and Reviews), 42(3), 408-420.

17. Liu, X. (2015). An optimal-distance-based transmission strategy for lifetime maximization of wireless sensor networks. IEEE Sensors Journal, 15(6), 3484-3491.

18. Vaishali, G., \& Nighot, M. K. (2016, August). An efficient ACO scheme for mobile-sink based WSN. In Inventive Computation Technologies (ICICT), International Conference on (Vol. 3, pp. 1-5). IEEE.

19. Dina S. Deif, \& Yasser Gadallah. (2017). An Ant Colony Optimization Approach for the Deployment of Reliable Wireless Sensor Networks, IEEE Access.

20. Liu, X. (2015). An optimal-distance-based transmission strategy for lifetime maximization of wireless sensor networks. IEEE Sensors Journal, 15(6), 3484-3491. 